\title{
An Individual Risk Model and Its Uncertainty Distribution
}

\author{
Li Ren* \\ Dongchang College of Liaocheng University, Liaocheng, China
}

(Received: September 5, 2012 / Revised: January 2, 2013 / Accepted: March 5, 2013)

\begin{abstract}
In insurance statistics, the sum of homogeneous insurance is often needed. The sum is called individual risk model which is a fundamental model in risk analysis for insurance. This paper first presents an individual risk model based on the uncertainty theory. Then its uncertainty distribution is provided. Finally, its arithmetic is shown by a numerical example.
\end{abstract}

Keywords: Uncertainty Theory, Individual Risk Model, Uncertainty Distribution

* Corresponding Author, E-mail: Lcjlk315@163.com

\section{INTRODUCTION}

It is well known that there are many types of uncertainty in real life. A. N. Kolmogoroff (1933) presented the probability theory dealing with a type of uncertainty that an event will happen (called randomness). Besides, Zadeh (1965) initializedthe fuzzy set theory via membership function that aimed to study the uncertainty called fuzzyness. The probability theory and the fuzzy set theory have been widely accepted to study uncertainness and have also been applied to many aspects of our life.

However, there are still many other uncertainness that can not be described by the probability theory and the fuzzy set theory. In order to rationally deal with the degree of belief, an uncertainty theory was introduced by Liu (2007). The uncertainty theory is a branch of axiomatic mathematics based on normality, duality, subadditivity and product axioms. And it was refined by Liu (2010a). Nowadays, the uncertainty theory has become a branch of axiomatic mathematics for modeling human uncertainty. Uncertain statistics is an important branch for uncertainty theory. It was introduced by Liu (2010a). Chen and Ralescu (2012) stated that the questionnaire survey may successfully acquire the expert's experimental data. Wang et al., (2012) recast the Delphi method as a process to determine the uncertainty distributions. Liu (2010a) suggested the principle of least squares method to propose an uncertainty distribution. Furthermore, the uncertainty theory has been applied to uncertain programming (Liu, 2009a; Gao, 2011, 2012; Meng and Zhang, 2013; Peng and Yao, 2011; Rong, 2011; Sheng and Yao, 2012; Zhang and Chen, 2012; Zhang and Meng, 2013), uncertain risk analysis (Liu, 2010b), uncer-tain logic (Chen et al., 2012; Li and Liu, 2009; Zhang, 2011; Zhang and Peng, n.d.) uncertain process (Yao and $\mathrm{Li}, 2012$ ), and others (Dai and Chen, 2012; Chen and Ralescu, 2011; Gao, 2009; Zhang et al., 2013; Zhu, 2010).

In order to study dynamic systems in uncertainty theory, Liu (2009b) introduced uncertain process which is essentially a sequence of uncertain variables indexed by time. There is an important type of uncertain process called renewal process, which is an uncertain process in which events occur continuously and independently of one another in uncertain times. Yao and Li (2012) studied the uncertain alternating renewal process. Based on the uncertain renewal process, Yao and Ralescu (2013) investigated the age replacement policy in uncertain environment. Liu (2013) presented an uncertain insurance model by assuming that the claim is an uncertain renewal reward process, and proved a formula for calcula- 
ting ruin index. Different from it, this paper aims to study insurance by uncertain statistics. We will build a flow shop scheduling model in the uncertain environment based on the uncertainty theory. It is well known that in insurance statistics the sum of homogeneous insurance is often needed, and the sum is called individual risk model, which is a fundamental model in risk analysis for insurance. These problems are studied by many scholars (Kass et al., 2001; Peng, 2009; Wen and Wang, 2006).

The paper is organized as follows. Section 2 recalls several concepts of uncertain variables. Section 3 introduces an individual risk model and its uncertainty distribution. Section 4 gives a numerical example. Finally, a brief summary is included.

\section{PRELIMINARIES}

In this section, we introduce some foundational concepts and properties of uncertainty theory, which will be used throughout this paper.

Let $\Gamma$ be a nonempty set, and $L$ a $\sigma$-algebra over $\Gamma$. Each element $\Lambda \in L$ is called an event.

Definition 1 (Liu, 2007). The set function $M$ is called an uncertain measure if it satisfies the following three axioms:

Axiom 1 (Normality). $M\{\Gamma\}=1$;

Axiom 2 (Self-Duality). $M\{\Lambda\}+M\left\{\Lambda^{c}\right\}=1$ for any event $\Lambda$;

Axiom 3 (Countable Subadditivity). For every countable sequence of events $\left\{\Lambda_{i}\right\}$, we have

$$
M\left\{\bigcup_{i=1}^{\infty} \Lambda_{i}\right\} \leq \sum_{i-1}^{\infty} M\left\{\Lambda_{i}\right\}
$$

Liu (2009b) presented the product measure axiom of uncertainty theory in 2009 as follows:

Axiom 4 (Product Measure Axiom). Let $\Gamma_{k}$ be nonempty sets on which $M_{k}$ are uncertain measures, $k=1,2, \cdots, n$, respectively. Then the product uncertain measure $M$ is an uncertain measure on the product $\sigma$-algebra $Ł_{1} \times Ł_{2} \times$ $\cdots \times Ł_{n}$ satisfying

$$
M\left\{\prod_{i=1}^{n} \wedge_{k}\right\}=\min _{1 \leq k \leq n} M_{k}\left\{\wedge_{k}\right\}
$$

That is, for each event $\Lambda \in €$, we have

$$
M\{\wedge\}=\left\{\begin{array}{c}
\sup _{\wedge_{1} \times \wedge_{2} \times \cdots \times \wedge_{n} \subset \wedge} \min _{1 \leq k \leq n} M_{k}\left\{\wedge_{k}\right\} \\
\text { if } \sup _{\wedge_{1} \times \wedge_{2} \times \cdots \times \wedge_{n} \subset \wedge} \min _{1 \leq k \leq n} M_{k}\left\{\wedge_{k}\right\}>0.5 \\
1-\sup _{\wedge_{\wedge_{1} \times \wedge_{2} \times \cdots \times \wedge_{n} \subset \wedge} \min _{c 1 \leq k \leq n} M_{k}\left\{\wedge_{k}\right\}} \\
\text { if } \sup _{\wedge_{1} \times \wedge_{2} \times \cdots \times \wedge_{n} \subset \wedge} \min _{c 1 \leq k \leq n} M_{k}\left\{\wedge_{k}\right\}>0.5 \\
0.5 \text { otherwise. }
\end{array}\right.
$$

Definition 2 (Liu, 2010a). An uncertain variable is a measurable function $\xi$ from an uncertainty space $(\Gamma, L$, $M)$ to the set of real numbers, i.e., for any Borel set $B$ of real numbers, the set

$$
\{\xi \in B\}=\{\gamma \in \Gamma \mid \xi(\gamma) \in B\}
$$

is an event.

Definition 3 (Liu, 2010a). The uncertainty distribution $\Phi$ of an uncertain variable $\xi$ is defined by

$$
\Phi(x)=M\{\xi \leq x\}
$$

for any real number $x$.

Definition 4 (Liu, 2010a). An uncertain variable $\xi$ is called linear if it has a linear uncertainty distribution

$$
\Phi(x) \begin{cases}0, & \text { if } x \leq a \\ (x-a) /(b-a), & \text { if } a \leq x \leq b \\ 1, & \text { if } x \geq b\end{cases}
$$

denoted by $\mathrm{\iota}(a, b)$ where $\mathrm{a}$ and $\mathrm{b}$ are real numbers with $a<b$.

Definition 5 (Liu, 2010a). An uncertain variable $\xi$ is called zigzag if it has a zigzag uncertainty distribution

$$
\Phi(x)= \begin{cases}0, & \\ (x-a) / 2(b-a), & \text { if } a \leq x \leq b \\ (x+c-2 b) / 2(c-b), & \text { if } b \leq x \leq c \\ 1, & \text { if } x \geq c\end{cases}
$$

denoted by $\boldsymbol{Z}(a, b, c)$ where a, b, c are real numbers with $a<b<c$.

Definition 6 (Liu, 2010a). An uncertain variable $\xi$ is called normal if it has a normal uncertainty distribution

$$
\Phi(x)=\left(1+\exp \left(\frac{\pi(e-x)}{\sqrt{3} \sigma}\right)\right)^{-1}
$$

denoted by $N(e, \sigma)$ where $e$ and $\sigma$ are real numbers with $\sigma>0$.

Definition 7 (Liu, 2010a). An uncertain variable $\xi$ is called lognormal if $\ln \xi$ is a normal uncertainty variable $N(e, \sigma)$. In other words, a lognormal uncertain variable has an uncertainty distribution

$$
\Phi(x)=\left(1+\exp \left(\frac{\pi(e-\ln x)}{\sqrt{3} \sigma}\right)\right)^{-1}
$$

denoted by $\operatorname{LOGN}(e, \sigma)$ where $e$ and $\sigma$ are real num- 
bers with $\sigma>0$.

Definition 8 (Liu, 2010a). The uncertain variables $X_{1}$, $X_{2}, \cdots, X_{m}$ are said to be independent if

$$
M\left\{\bigcap_{i=1}^{m} X_{i} \in B_{i}\right\}=\min _{1 \leq i \leq m} M\left\{X_{i} \in B_{i}\right\}
$$

for any Borel sets $B_{1}, B_{2}, \cdots, B_{m}$ of real numbers.

Definition 9 (Liu, 2010a). An uncertainty distribution $\Phi$ is said to be regular if its inverse function $\Phi^{-1}(\alpha)$ exists and is unique for each $\alpha \in(0,1)$.

Theorem 1 (Liu, 2010a). Let $\xi_{1}, \xi_{2}, \cdots, \xi_{n}$ be independent uncertain variables with uncertainty distributions $\Phi_{1}, \Phi_{2}, \cdots, \Phi_{n}$, respectively. If $f$ is a strictly increasing function, then

$$
\xi=f\left(\xi_{1}, \xi_{2}, \cdots, \xi_{n}\right)
$$

is an uncertain variable with inverse uncertainty distribution

$$
\Psi^{-1}(\alpha)=f\left(\Phi_{1}^{-1}(\alpha), \Phi_{2}^{-1}(\alpha), \cdots, \Phi_{n}^{-1}(\alpha)\right) .
$$

Theorem 2 (Liu, 2010a). Let $\xi_{1}, \xi_{2}, \cdots, \xi_{n}$ be independent uncertain variables with uncertainty distributions $\Phi_{1}, \Phi_{2}, \cdots, \Phi_{n}$, respectively. If $f$ is a strictly increasing function, then

$$
\xi=f\left(\xi_{1}, \xi_{2}, \cdots, \xi_{n}\right)
$$

is an uncertain variable with inverse uncertainty distribution

$$
\Psi(x)=\sup _{f\left(x_{1}, x_{2}, \cdots, x_{n}\right)} \min _{1 \leq i \leq n} \Phi_{i}\left(x_{i}\right) .
$$

\section{AN INDIVIDUAL RISK MODEL AND ITS UNCERTAINTY DISTRIBUTION}

In this section, we will present an individual risk model based on the uncertainty theory. It is a fundamental model in risk analysis for insurance. Assume that in a stated time (such as one year's time), the insurer has done business with $n$ customers, and every customer bought $N$ policies. It is well known that in an insurance company, if customers have some prescriptive losses, then the insurer should shell out the payment. It is evident that the payment is an uncertain variable. Thus, we have the the following definition:

Definition 10. In a stated time, the total indemnity for the insurer is given by

$$
S=\sum_{j=1}^{N} \sum_{i=1}^{n_{j}} \xi_{i j},
$$

which is called individual risk model, where $\xi_{i j}$ are the payment for the $j$ th insurance contract of $i$ th insured, and $n_{j}$ customers subscribe the $j$ th insurance contract.

The quantity of the insurance contracts is supplied by the insurer.

Commonly, it is hard to obtain the accurate distribution of $S$. In order to study the distribution of $S$ and obtain its properties, some hypotheses should be given.

1) The uncertainty distribution $\varphi_{i j}$ of every uncertain variable $\xi_{i j}$ are as follows:

$$
\varphi_{i j}(x)=M\left\{\xi_{i j} \leq x\right\}= \begin{cases}0, & \text { if } x<0 \\ p(x), & \text { if } 0 \leq x<b \\ 1, & \text { if } x \geq b,\end{cases}
$$

where 0 is the minimum payment, $b$ is the maximum payment and $p(x)$ is a strictly increasing function.

2) Uncertain variables $\xi_{i j}$ are independent.

3) Every policy settles a claim once at most.

4) The homogeneous insurances have characteristic of the same insurance marks. From the thought of uncertainty theory, this kind of homogeneity shows that the payment variables $\xi_{i j}$ of the same kind insurance policy are iid uncertain variables. This means

$$
\varphi_{1 j}(x)=\varphi_{2 j}(x)=\varphi_{3 j}(x)=\cdots=\varphi_{n_{j}}(x) .
$$

Based on the above hypothesis, we give the distribution of $S$ in the following.

Theorem 3. In the individual risk model with the limit of $N=1$, then $S=\sum_{i=1}^{n} \xi_{i}$ is an uncertain variable with the uncertainty distribution

$$
\Phi(x)=\varphi\left(\frac{x}{n}\right),
$$

and its inverse uncertainty distribution

$$
\Phi^{-1}(\alpha)=n \varphi^{-1}(\alpha),
$$

where $\xi_{1}, \xi_{2}, \cdots, \xi_{n}$ are independent uncertain variables with the common uncertainty distribution $\varphi(x)$.

Proof. Note that $\xi_{1}, \xi_{2}, \cdots, \xi_{n}$ are independent uncertain variables with the common uncertainty distribution $\varphi(\alpha)$. By Theorem 2, we have

$$
\Phi(x)=\sup _{x_{1}+x_{2}+\cdots+x_{n}=x} \min _{1 \leq i \leq n} \varphi\left\{x_{i}\right\} .
$$

It is evident that $n \times \frac{x}{n}=x$ and

$$
\varphi\left(\frac{x}{n}\right) \wedge \varphi\left(\frac{x}{n}\right) \wedge \cdots \wedge \varphi\left(\frac{x}{n}\right)=\varphi\left(\frac{x}{n}\right) .
$$


Counterevidence. Suppose $\Phi(x) \neq \varphi\left(\frac{x}{n}\right)$. If $\Phi(x)>$ $\varphi\left(\frac{x}{n}\right)$, then there exists $x_{1}, x_{2}, \cdots, x_{n}$ such that $x_{1}+x_{2}+$ $\cdots+x_{n}=x$ and $\varphi\left(x_{i}\right)>\varphi\left(\frac{x}{n}\right), i=1,2, \cdots, n$. Since $\varphi(x)$ is a increasing function, $x_{2}>\frac{x}{n}$, this is inconsistent with $x_{1}+x_{2}+\cdots+x_{n}=x$. Thus

$$
\Phi(x)=\varphi\left(\frac{x}{n}\right) .
$$

Since $S=\sum_{i=1}^{n} \xi_{i}$ and it increase on each $\xi_{i}, i \in 1,2, \cdots$, $n$, by Theorem 1 we have

$$
\Phi^{-1}(\alpha)=\varphi^{-1}(\alpha)+\varphi^{-1}(\alpha)+\cdots+\varphi^{-1}(\alpha)=n \varphi^{-1}(\alpha) .
$$

The theorem is proved.

Theorem 4. In the above individual risk model, $S=$ $\sum_{j=1}^{N} \sum_{i=1}^{n_{j}} \xi_{i j}$ is an uncertain variable with the uncertainty distribution

$$
\Psi(x)=\sup _{x_{1}+x_{2}+\cdots+x_{N}=} \min _{1<j \leq N} \Phi_{j}\left(\frac{x_{j}}{n_{j}}\right),
$$

and its inverse uncertainty distribution is

$$
\Psi^{-1}(\alpha)=\sum_{j=1}^{N} n_{j} \Phi_{j}^{-1}(\alpha)
$$

where $\Phi_{j}\left(x_{j}\right)=M\left\{\xi_{i j}<x_{j}\right\}, i \in\left\{1,2, \cdots, n_{j}\right\}, 0<j \leq N$.

Proof. Let

$$
\xi_{j}=\sum_{i=1}^{n_{j}} \xi_{i j}
$$

Note that $\xi_{1 j}, \xi_{2 j}, \cdots, \xi_{n_{j} j}$ are independent uncertain variables with the common uncertainty distribution $\varphi(x)$. Since $\Phi_{j}(x)=M\left\{\xi_{i j}<x_{j}\right\}, i \in\left\{1,2, \cdots, n_{j}\right\}, 0<j \leq N$, by Theorem 3, we have $\Phi_{j}\left(x_{j}\right)=\varphi_{j}\left(\frac{x_{j}}{n}\right)$. Since $S=\sum_{j=1}^{N} \sum_{i=1}^{n_{j}} \xi_{i j}$ $=\sum_{j=1}^{N} \xi_{j}$, by Theorem 2, we have

$$
\psi(x)=\sup _{x_{1}+x_{2}+\cdots x_{N}=x} \min _{0<j \leq N} \Phi_{j}\left(\frac{x_{j}}{n_{j}}\right) .
$$

By Theorem 3 and Theorem 1, inverse uncertainty distribution of $S=\sum_{j=1}^{N} \sum_{i=1}^{n_{j}} \xi_{i j}=\sum_{j=1}^{N} \xi_{j}$ is

$$
\psi^{-1}(\alpha)=\sum_{j=1}^{N} n_{j} \Phi_{j}^{-1}(\alpha)
$$

The theorem is proved.

\section{A NUMERICAL EXAMPLE}

Assume that the insurers have sold four kinds of policies to a fellowship with 1,000 members, and the distributions of these policies are as follows,

$$
\begin{aligned}
& \varphi_{i 1}(x)=M\left\{\xi_{i 1} \leq x\right\}= \begin{cases}0, & \text { if } x<0 \\
x, & \text { if } 0 \leq x<2 \\
1, & \text { if } x \geq 2,\end{cases} \\
& \varphi_{i 2}(x)=M\left\{\xi_{i 2} \leq x\right\}= \begin{cases}0, & \text { if } x<0 \\
x / 2, & \text { if } 0 \leq x<1 \\
(x+1) / 4, & \text { if } 1 \leq x \leq 3 \\
1, & \text { if } x \geq 3,\end{cases} \\
& \varphi_{i 3}(x)=M\left\{\xi_{i 3} \leq x\right\}= \begin{cases}0, & \text { if } x<0 \\
(1+\exp (\pi(2-x)))^{-1}, & \text { if } 0 \leq x<4 \\
1, & \text { if } x \geq 4,\end{cases} \\
& \varphi_{i 4}(x)=M\left\{\xi_{i 4} \leq x\right\}= \begin{cases}0, & \text { if } x<0 \\
\left(1+\exp \left(\frac{\pi(1-\ln x)}{2}\right)\right)^{-1}, & \text { if } 0 \leq x<5 \\
1, & \text { if } x \geq 5 .\end{cases}
\end{aligned}
$$

Then by the distribution of it we can get the uncertainty distribution for the total payments lacked in the coming year as follow:

$$
\begin{aligned}
\Psi(x)= & \sup _{x_{1}+x_{2}+x_{3}+x_{4}=x} \varphi_{i 1}\left(\frac{x_{1}}{1000}\right) \wedge \varphi_{i 2}\left(\frac{x_{2}}{1000}\right) \\
& \wedge \varphi_{i 3}\left(\frac{x_{3}}{1000}\right) \wedge \varphi_{i 4}\left(\frac{x_{4}}{1000}\right) \\
= & \begin{cases}0, & \text { if } x<0 \\
\left(1+\exp \left(\frac{\pi(1-\ln x)}{2}\right)\right)^{-1}, & \text { if } 0 \leq x<14000 \\
1, & \text { if } x \geq 14000 .\end{cases}
\end{aligned}
$$

\section{CONCLUSION}

This paper provided an individual risk model via uncertainty theory and gave its uncertainty distribution. The model and its arithmetic were shown by a numerical example.

\section{ACKNOWLEDGMENTS}

This work was supported by National Natural Science Foundation of China (Grant No. 61273044). 


\section{REFERENCES}

Chen, X. and Ralescu, D. A. (2011), A note on truth value in uncertain logic, Expert Systems With Applications, 38(12), 15582-15586.

Chen, X. and Ralescu, D. A. (2012), B-spline method of uncertain statistics with application to estimating travel distance, Journal of Uncertain Systems, 6(4), 256-262.

Chen, X., Kar, S., and Ralescu, D. A. (2012), Crossentropy measure of uncertain variables, Information Sciences, 201, 53-60.

Dai, W. and Chen, X. (2012), Entropy of function of uncertain variables, Mathematical and Computer Modelling, 55(3/4), 754-760.

Gao, X. (2009), Some properties of continuous uncertain measure, International Journal of Uncertainty, Fuzziness and Knowledge-Based Systems, 17(3), 419-426.

Gao, Y. (2011), Shortest path problem with uncertain arc lengths, Computers and Mathematics with Applications, 62(6), 2591-2600.

Gao, Y. (2012), Uncertain models for single facility location problem on networks, Applied Mathematical Modelling, 36(6), 2592-2599.

Kass, R., Goovaerts, M., Dhaene, J., and Denuit, M. (2001), Modern Actuarial Risk Theory, Kluwer Academic Publishers, New York, NY.

Kolmogorov, A. N. (1933), Grundbegriffe der Wahrscheinlichkeitsrechnung, Julius Springer, Berlin.

Li, X. and Liu, B. (2009), Hybrid logic and uncertain logic, Journal of Uncertain Systems, 3(2), 83-94.

Liu, B. (2007), Uncertainty Theory (2nd ed.), SpringerVerlag, Berlin.

Liu, B. (2009a), Theory and practice of uncertain programming (2nd ed.), Springer-Verlag, Berlin.

Liu, B. (2009b), Some research problems in uncertainty theory, Journal of Uncertain Systems, 3(1), 3-10.

Liu, B. (2009), Some research problems in uncertainty theory, Journal of Uncertain Systems, 3(1), 3-10.

Liu, B. (2010a), Uncertainty Theory: A Branch of Mathematics for Modeling Human Uncertainty, SpringerVerlag, Berlin.

Liu, B. (2010b), Uncertain risk analysis and uncertain reliability analysis, Journal of Uncertain Systems, 4(3), 163-170.

Liu, B. (2013), Extreme value theorems of uncertain process with application to insurance risk model, Soft Computing, 17(4), 549-556.

Meng, G. and Zhang, X. (2013), Optimization uncertain measure model for uncertain vehicle routing pro- blem, Information, 16(2), 1201-1206.

Peng, J. (2009), Value at risk and tail value at risk in uncertain environment, Proceedings of the 8th International Conference on Information and Management Sciences, Kunming, China, 787-793.

Peng, J. and Yao, K. (2011), A new option pricing model for stocks in uncertainty markets, International Journal of Operations Research, 8(2), 18-26.

Rong, L. (2011), Two new uncertainty programming models of inventory with uncertain costs, Journal of Information and Computational Science, 8(2), 280-288.

Sheng, Y. and Yao, K. (2012), Fixed charge transportation problem and its uncertain programming model, Industrial Engineering and Management Systems, 11(2), 183-187.

Wang, X., Gao, Z., and Guo, H. (2012),Uncertain hypothesis testing for two experts' empirical data, Mathematical and Computer Modelling, 55(3/4), 14781482.

Wen, L. and Wang, Y. (2006), Risk Theory, Finance Economy Publishing Company, Beijing.

Yao, K. and Li, X. (2012), Uncertain alternating renewal process and its application, IEEE Transactions on Fuzzy Systems, 20(6), 1154-1160.

Yao, K. and Ralescu, D. A. (2013), Age replacement policy in uncertain environment, Iranian Journal of Fuzzy Systems, 10(2), 29-39.

Zadeh, L. A. (1965), Fuzzy sets, Information and Control, 8, 338-353.

Zhang, X. (2011), Duality and pseudo duality of dual disjunctive normal forms, Knowledge-Based Systems, 24(7), 1033-1036.

Zhang, X. and Chen, X. (2012), A new uncertain programming model for project problem, Information, 15(10), 3901-3910.

Zhang, X. and Meng, G. (2013), Expected-varianceentropy model for uncertain parallel machine scheduling, Information, 16(2), 903-908.

Zhang, X. and Peng, Z. (n.d.), Uncertain predicate logic based on uncertainty theory, http://orsc.edu.cn/on line/091204. pdf.

Zhang, X., Ning, Y., and Meng, G. (2013), Delayed renewal process with uncertain interarrival times, Fuzzy Optimizationand Decision Making, 12(1), 79-87.

Zhu, Y. (2010), Uncertain optimal control with application to a portfolio selection model, Cybernetics and Systems, 41(7), 535-547. 